\title{
The Clinical Impact of 6-Min Walk Test Distance as a Predictor of Complications in Patients Undergoing Major Surgery
}

\author{
Kassem Kassak $^{1} \cdot$ Ahmad Husari $^{2,3}$
}

Published online: 25 August 2015

(C) Société Internationale de Chirurgie 2015

Significantly reduced 6-min walk test (6MWT) distances, in our opinion, reflect limited functional health status of patients undergoing the test. This is an additional tool for patients and surgeons to consider in deciding whether to proceed with major surgical procedures or opt for medical treatment. The poor 6MWT results may also swing physicians to abandon, altogether, open traditional surgeries in favor of newer technologies/procedures. In a study by Bauger et al., 6MWT was performed on patients with severe aortic stenosis who were considered a very high surgical risk for traditional approach [1]. The mean 6MWT distance walked was $165.3 \pm 79.7 \mathrm{~m}$ which reflected the health status of those high-risk patients. Similarly on examining our data, we noted that a 6MWT distance of 250 m may be a "cut-off distance" below which the surgical risks and complications are significantly increased. Surgeons should deliberate the findings of reduced 6MWT

All authors have read and approved submission of the manuscript and the manuscript has not been published and is not being considered for publication elsewhere in whole or part in any language except as an abstract.

Ahmad Husari

ah51@aub.edu.lb

1 Department of Health Management and Policy, American University of Beirut-Medical Center, P.O. Box: 113-6044, Beirut 1107 2802, Lebanon

2 Division of Pulmonary and Critical Care Medicine, Department of Internal Medicine, American University of Beirut-Medical Center, P.O. Box: 113-6044, Beirut 1107 2802, Lebanon

3 Department of Internal Medicine, Faculty of Medicine, American University of Beirut Medical Center, Riad El Solh, P.O. Box 11-236, Beirut 1107 2020, Lebanon distances $(<250 \mathrm{~m})$ very seriously with their patients before proceeding with surgery. Again, alternative approaches should be considered. In the case of severe aortic stenosis, for example, transcatheter aortic valve implantation should be considered first rather than proceeding with traditional open thoracotomy surgery.

In reference to the linear regression model adopted in this study, it actually included all independent variables, and only those that demonstrated significance $(P<0.05)$ were reported in the results section. Finally, we totally agree with Straatman et al., algorithms in assessing patients preoperatively and in the immediate $24 \mathrm{~h}$, which are essential tools in improving health care delivery, outcomes, and cost containment [2].

\section{References}

1. Bagur R, Rodés-Cabau J, Dumont E, Larochellière RD, Doyle D, Bertrand OF, Côté M, Poirier P, Pibarot P (2011) Exercise capacity in patients with severe symptomatic aortic stenosis before and 6 months after transcatheter aortic valve implantation. Am J Cardiol 108(2):258-264

2. Husari AW, Belzberg H, Kassak K, Dunham CM (2009) Relationship between intensive care complications and costs and initial $24 \mathrm{~h}$ events of trauma patients with severe haemorrhage. Emerg Med J 5:340-343 